\title{
An Extrinsic-Inductance Independent Approach for Direct Extraction of HBT Intrinsic Circuit Parameters
}

\author{
Tzyy-Sheng Horng, Member, IEEE, Jian-Ming Wu, and Hui-Hsiang Huang
}

\begin{abstract}
A novel analytical procedure has been proposed for direct extraction of the intrinsic elements in a hybrid- $\pi$ equivalent circuit of heterojunction bipolar transistors. This method differs from previous ones by formulating impedance-parameter based expressions that are exclusive of the extrinsic inductances associated with the base, emitter, and collector. It is therefore not susceptible to variation of the extrinsic reactances from dc to high frequencies and can lead to very accurate extraction of the intrinsic elements under different bias conditions. The distributed phenomena in the base region can be also characterized rigorously by exploiting the bias-independent features of the extrinsic elements that are extracted subsequently from knowledge of the intrinsic elements.
\end{abstract}

Index Terms-Heterojunction bipolar transistors, parameter extraction, semiconductor device modeling.

\section{INTRODUCTION}

$\mathbf{I}$ $\mathrm{N}$ CONSIDERATION of efficiency and uniqueness, direct extraction approaches have been intensively used for modeling heterojunction bipolar transistors (HBTs) [1]-[6]. All approaches without exception follow that the extrinsic elements are first determined and then de-embedded to allow for extraction of the intrinsic elements. The reported extraction methods for extrinsic inductances rely on either test structure measurements [1] or forward-biased measurements under high base currents [2]-[6]. However, there are still some empirical difficulties in both techniques. The former technique requires separate measurement of $S$-parameters in a shorted test structure with an identical interconnection pattern in a real device. To evaluate the parasitic inductances effectively, the interconnection pattern is usually designed simply but not practically enough for modeling multiple HBTs in an array form for power amplifier applications. The latter technique takes the $S$-parameter measurement when HBTs' base-collector and base-emitter junctions are maintained forward-biased. The injected base current densities have to be sufficiently high so as to make the junction impedances negligible when compared to the extrinsic impedances. Under this scheme, the extrinsic inductances are better extracted with larger reactances at higher frequencies. However, the range of base-current density and frequency for

Manuscript received March 29, 2001; revised August 21, 2001. This work was supported by the National Science Council, Taiwan R.O.C., under Grant NSC-89-2215-E-110-023.

The authors are with the Department of Electrical Engineering, National Sun Yat-Sen University, Kaohsiung 804, Taiwan, R.O.C. (e-mail: jason@ ee.nsysu.edu.tw).

Publisher Item Identifier S 0018-9480(01)10465-5.

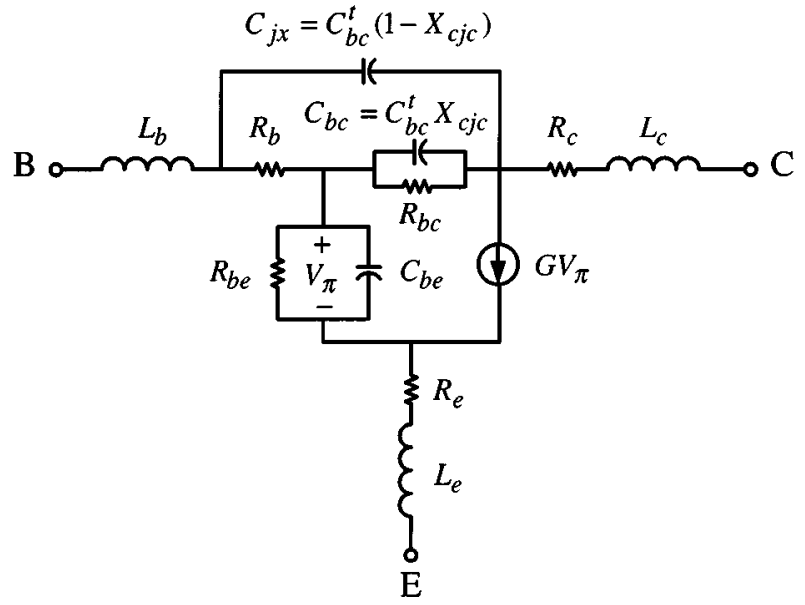

Fig. 1. Gummel-Poon small-signal model for an npn AlGaAs-GaAs HBT.

a robust extraction of extrinsic inductances cannot normally be forecast unless intensive tests have been carried out for a while. To save strength in extracting extrinsic inductances but still have reliable extracted quantities for intrinsic elements, our formalism is quite unique to be able to express each intrinsic element in a mathematical form independent of all extrinsic inductances [7]. In [6], an extrinsic-inductance insensitive expression for extracting the base resistance has been proposed as a starting point for extracting the intrinsic elements. The entire formulation is still extrinsic-inductance-dependent and at least needs some rough figures for all extrinsic inductances before extracting the intrinsic elements.

Fig. 1 shows the Gummel-Poon small-signal model of an npn AlGaAs-GaAs HBT in consideration of negligible substrate effects. The coefficient $X_{c j c}$ with quantity between $0-1$ represents the splitting in the base-collector junction capacitance to account for the distributed effects at high frequencies in the base region. Such distributed base effects complicate the formulation to make the direct parameter extraction more cumbersome. The approaches in [2], [4], and [5] employ full knowledge of the extracted extrinsic elements to de-embed the intrinsic network parameters whose frequency dependence can determine the coefficient effectively. However, the calculated coefficients have been found to be quite sensitive to the extrinsic inductances that were extracted beforehand. The formulation in [6] is less sensitive to variation of the inductance quantities but requires extraction of the base resistance in advance from the $S$-parameters measured at sufficiently high frequencies, which often go to millimeter-wave range for smaller HBT devices. In this paper, 
the extrinsic emitter inductance has been found to have a tight dependence on the coefficient and their mathematical relation has been derived skillfully. We can further exploit bias independence of the emitter inductance to quantify precisely this coefficient.

\section{EXTRACTION OF INTRINSIC ELEMENTS}

By referring to [6], the two-port impedance parameters for the HBT's hybrid- $\pi$ equivalent circuit as shown in Fig. 1 are derived as

$$
\begin{aligned}
Z_{11}= & j \omega\left(L_{b}+L_{e}\right)+R_{e}+\frac{Z_{b e}}{1+G Z_{b e}}+\frac{R_{b} Z_{j x}}{R_{b}+Z_{b c}+Z_{j x}} \\
& +\frac{Z_{b c} R_{b}}{\left(1+G Z_{b e}\right)\left(R_{b}+Z_{b c}+Z_{j x}\right)} \\
Z_{12}= & j \omega L_{e}+R_{e}+\frac{Z_{b e}}{1+G Z_{b e}} \\
& +\frac{Z_{b c} R_{b}}{\left(1+G Z_{b e}\right)\left(R_{b}+Z_{b c}+Z_{j x}\right)} \\
Z_{21}= & j \omega L_{e}+R_{e}+\frac{Z_{b e}}{1+G Z_{b e}} \\
& +\frac{Z_{b c} R_{b}-G Z_{b e} Z_{b c} Z_{j x}}{\left(1+G Z_{b e}\right)\left(R_{b}+Z_{b c}+Z_{j x}\right)} \\
Z_{22}= & j \omega\left(L_{e}+L_{c}\right)+R_{e}+R_{c}+\frac{Z_{b e}}{1+G Z_{b e}} \\
& +\frac{Z_{b c}\left(R_{b}+Z_{j x}\right)}{\left(1+G Z_{b e}\right)\left(R_{b}+Z_{b c}+Z_{j x}\right)}
\end{aligned}
$$

where $Z_{b e}=\left(g_{b e}+j \omega C_{b e}\right)^{-1}, Z_{b c}=\left(g_{b c}+j \omega C_{b c}\right)^{-1}, Z_{j x}=$ $\left(j \omega C_{j x}\right)^{-1}$, and $G=g_{m} e^{-j \omega \tau}$. We rearrange the formulation to find the extrinsic-inductance independent expressions given as

$$
\begin{aligned}
Z_{12}-Z_{21} & =\frac{Z_{b c} Z_{j x}}{(1+U)\left(R_{b}+Z_{b c}+Z_{j x}\right)} \\
\operatorname{real}\left\{Z_{11}-Z_{12}\right\}= & \operatorname{real}\left\{\frac{R_{b} Z_{j x}}{R_{b}+Z_{b c}+Z_{j x}}\right\} \\
\operatorname{real}\left\{Z_{22}-Z_{12}\right\}= & R_{c}+\operatorname{real}\left\{\left(Z_{12}-Z_{21}\right) U\right\} \\
\operatorname{real}\left\{Z_{12}\right\}= & R_{e}+\operatorname{real}\left\{\frac{1}{G(1+U)}\right\} \\
& +\operatorname{real}\left\{\left(Z_{12}-Z_{21}\right) U \frac{R_{b}}{Z_{j x}}\right\}
\end{aligned}
$$

where

$$
U=\left(G Z_{b e}\right)^{-1}=\left(\beta^{-1}+j \omega \frac{C_{b e}}{g_{m}}\right) e^{j \omega \tau} .
$$

In (9), $\beta=g_{m} R_{b e}$ and $e^{j \omega \tau}$ can be further approximated by the first three terms in the Taylor expansion series

$$
e^{j \omega \tau} \approx 1+j \omega \tau-\frac{\omega^{2} \tau^{2}}{2}
$$

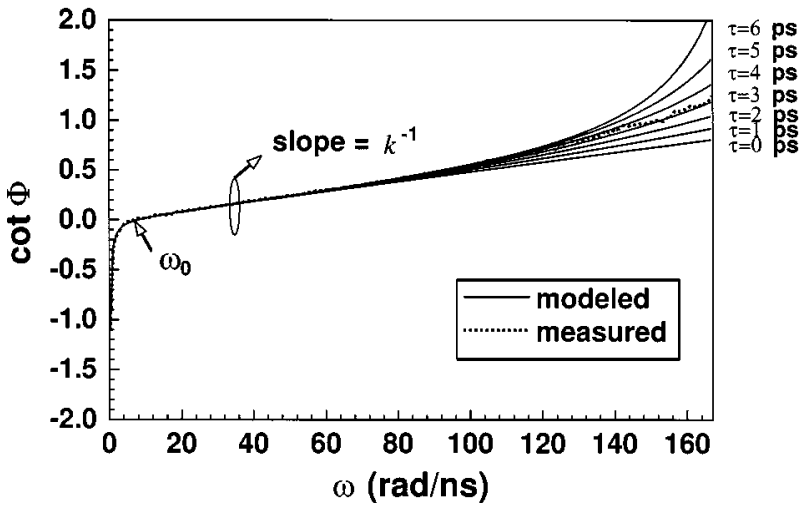

Fig. 2. Illustration of a curve-fit procedure for estimating the parameters $\omega_{0}$, $k$, and $\tau$.

Substituting (9) and (10) into (5), we inspect the resultant formulation and omit some negligible terms based on the following assumptions:

$$
\begin{gathered}
R_{b} \ll \min \left(R_{b c}, \frac{4 C_{b e}}{g_{m} C_{b c}^{t}}\right) \\
\frac{R_{b} C_{b c}^{t}}{2} \ll \tau \ll \min \left(R_{b e} C_{b e}, R_{b c} C_{b c}^{t}\right)
\end{gathered}
$$

where $C_{b c}^{t}$ represents the amount of $C_{b c}$ and $C_{j x}$. Note that the above assumptions are mostly good for an HBT operating in the forward active region. Finally, the equation in (5) is approximated as

$$
\begin{aligned}
Z_{12}-Z_{21} \approx\left(C_{b c}^{t}\right)^{-1}[ & \frac{C_{b e}}{g_{m}}\left(j \omega \frac{g_{b c}}{C_{b c}^{t}}-\omega^{2}-j \omega^{3} \tau+\frac{\omega^{4} \tau^{2}}{2}\right) \\
& \left.+\left(1+\beta^{-1}\right)\left(\frac{g_{b c}}{C_{b c}^{t}}+j \omega\right)\right]^{-1} \cdot
\end{aligned}
$$

The most crucial step in this extraction algorithm is to derive the phase of the difference between two mutual impedance parameters as

$$
\cot \Phi \equiv \cot \angle\left(Z_{12}-Z_{21}\right) \approx \frac{\omega^{2}-\omega_{0}^{2}-\frac{\omega^{4} \tau^{2}}{2}}{\omega k-\omega^{3} \tau}
$$

where

$$
\begin{aligned}
\omega_{0} & =\sqrt{\left(1+\beta^{-1}\right) \frac{g_{m} g_{b c}}{C_{b e} C_{b c}^{t}}} \\
k & =\left(1+\beta^{-1}\right) \frac{g_{m}}{C_{b e}}+\frac{g_{b c}}{C_{b c}^{t}} .
\end{aligned}
$$

In the extraction, the measured $S$-parameters are converted into the impedance parameters in which the cotangent of the phase angle for the difference between two mutual terms is calculated as a function of radial frequency, as shown in Fig. 2 for an npn AlGaAs-GaAs HBT with $2.4 \times 6 \mu \mathrm{m}^{2}$ emitter area and biased at $V_{c e}=3 \mathrm{~V}$ and $I_{c}=2.88 \mathrm{~mA}\left(J_{c}=20 \mathrm{kA} / \mathrm{cm}^{2}\right)$. The parameters $\omega_{0}$ and $k$ in (15) and (16) can be estimated from such a cotangent function by its root and the inverse of its slope at low radial frequencies, respectively. The delay time $(\tau)$ can be pinpointed by a match of the curvature at high radial frequencies. It is noted that these $S$-parameters were measured via an 


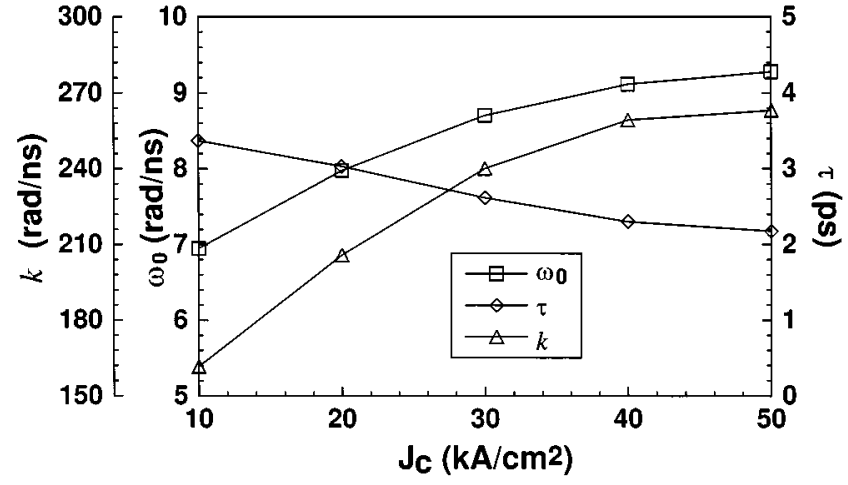

Fig. 3. The estimated quantities of the parameters $\omega_{0}, k$, and $\tau$ for the $2.4 \times$ $6 \mu \mathrm{m}^{2}$ emitter-area HBT biased at different collector current densities.

HP8510C network analyzer and Cascade Microtech probes with a frequency sweep from 0.5 to $26.5 \mathrm{GHz}$. The pad capacitances were calibrated in advance using the open pads. One caution is that the calibration errors in pad capacitances will lead eventually to the errors in extracting $\omega_{0}$ and $k$, and subsequently the intrinsic elements. This issue deserves special attention in modeling small-size HBTs when their junction capacitances are regarded as comparable to the pad capacitances. Once both $\omega_{0}$ and $k$ have been found, solving for $\tau$ directly from (14) yields a formula with much better accuracy in determining the quantity. That is,

$$
\tau \approx \frac{\cot \Phi}{\omega}-\frac{1}{\omega} \sqrt{\cot ^{2} \Phi-\frac{2 k}{\omega} \cot \Phi-2\left(\frac{\omega_{0}^{2}}{\omega^{2}}-1\right)} .
$$

As a result, the estimated quantities of $\omega_{0}, k$, and $\tau$ against the HBT's collector current density are plotted in Fig. 3 and will be used to extract the intrinsic elements in the next step.

From (15) and (16), one can solve for $g_{m} / C_{b e}$ and $g_{b c} / C_{b c}^{t}$ in terms of $\omega_{0}, k$, and $\beta$ and then substitute them into (6), (8), (9), and (13) to find the following intrinsic elements:

$$
\begin{aligned}
C_{b c}^{t} \approx \text { real } & \left\{( Z _ { 1 2 } - Z _ { 2 1 } ) ( 1 + \beta ^ { - 1 } ) \left[Q\left(\omega_{0}-\frac{\omega^{2}}{\omega_{0}}+\frac{\omega^{4} \tau^{2}}{2 \omega_{0}}\right)\right.\right. \\
& \left.\left.+j \omega\left(1+Q^{2}-\frac{Q \omega^{2} \tau}{\omega_{0}}\right)\right]\right\}^{-1}
\end{aligned}
$$

$R_{b c} \approx\left(\omega_{0} Q C_{b c}^{t}\right)^{-1}$

$R_{b} \approx \frac{\operatorname{real}\left\{Z_{11}-Z_{12}\right\}}{\operatorname{real}\left\{\left(Z_{12}-Z_{21}\right)(1+U)\left(\omega_{0} Q+j \omega X_{c j c}\right) C_{b c}^{t}\right\}}$

$g_{m} \approx \frac{\operatorname{real}\left\{e^{j \omega \tau}(1+U)^{-1}\right\}}{\operatorname{real}\left\{Z_{12}\right\}-R_{e}+\operatorname{imag}\left\{\left(Z_{12}-Z_{21}\right) U\right\} \omega R_{b} C_{b c}^{t}\left(1-X_{c j c}\right)}$

$C_{b e} \approx g_{m}\left(1+\beta^{-1}\right) \frac{Q}{\omega_{0}}$

where

$$
Q=\frac{1-\sqrt{1-\left(2 \omega_{0} k^{-1}\right)^{2}}}{2 \omega_{0} k^{-1}}
$$

and $U$ in (9) can be rewritten as

$$
U=\left[\beta^{-1}+j \omega \omega_{0}^{-1} Q\left(1+\beta^{-1}\right)\right] e^{j \omega \tau} .
$$

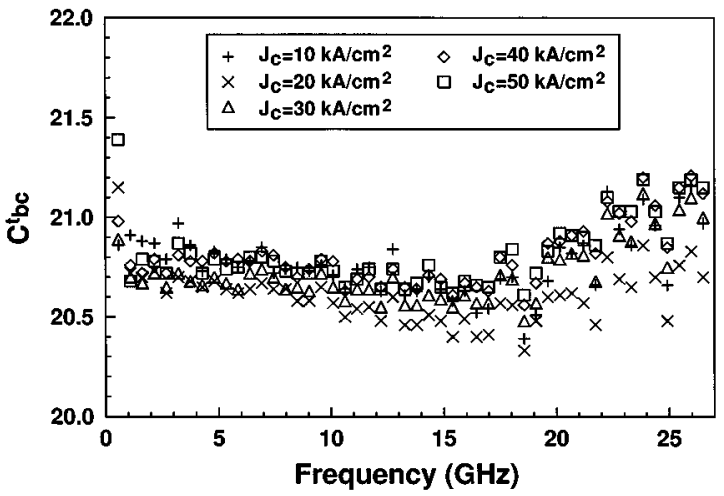

Fig. 4. The extracted quantities of the total base-collector capacitance for the $2.4 \times 6 \mu \mathrm{m}^{2}$ emitter-area HBT biased at different collector current densities.

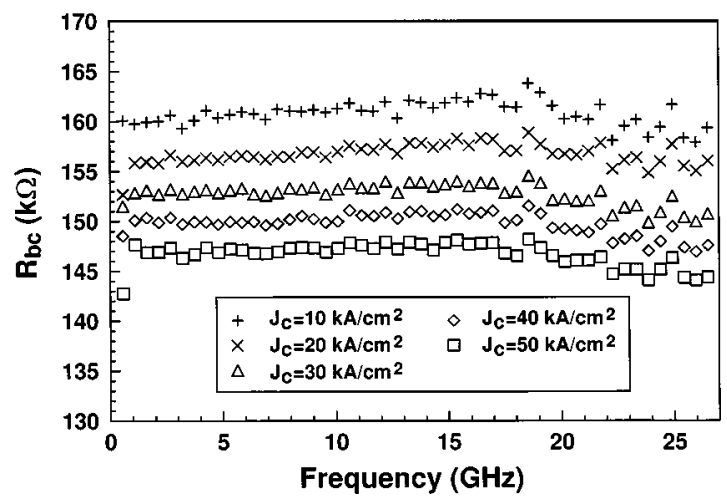

Fig. 5. The extracted quantities of the base-collector resistance for the $2.4 \times$ $6 \mu \mathrm{m}^{2}$ emitter-area HBT biased at different collector current densities.

In (18)-(22), the intrinsic elements are all expressed in terms of $\omega_{0}, k, \tau, \beta, X_{c j c}, R_{e}$ and the impedance parameters. There are still three unknowns $\beta, X_{c j c}, R_{e}$ to be determined before extracting the intrinsic elements. Generally speaking, these three parameters have a common feature of weak bias dependence. The current gain $\beta$ can be measured statically from the Gummel plots. In fact, the static value of $\beta$ is slightly different from the $\mathrm{RF}$ value, however, the influence due to the difference on the intrinsic elements except the base-emitter resistance $\left(R_{b e}\right)$ is quite small. The coefficient $X_{c j c}$ describing the nature of the distributed base can be estimated roughly from the HBT geometry. It was also examined in all intrinsic elements that only the base resistance $\left(R_{b}\right)$ shows a closer dependence on this coefficient. Therefore, it is not necessary to know both quantities of $\beta$ and $X_{c j c}$ to great precision for extracting the intrinsic elements, including $C_{b c}^{t}, R_{b c}, g_{m}$, and $C_{b e}$. The emitter resistance $\left(R_{e}\right)$ was found simply using the static flyback measurement [8]. For the HBT mentioned above, the quantities of $\beta, X_{c j c}$, and $R_{e}$ were estimated as $59,0.75$, and 10.1 respectively. The intrinsic elements $C_{b c}^{t}, R_{b c}, g_{m}$, and $C_{b e}$ can be then calculated from (18)-(22) against the measurement frequency from 0.5 to $26.5 \mathrm{GHz}$ under different collector current densities, as shown in Figs. 4-7. It is obvious to find that each intrinsic element exhibits a weak dependence on frequency in a variety of bias conditions, which implies a strong degree of accuracy and robustness for this extraction technique. 


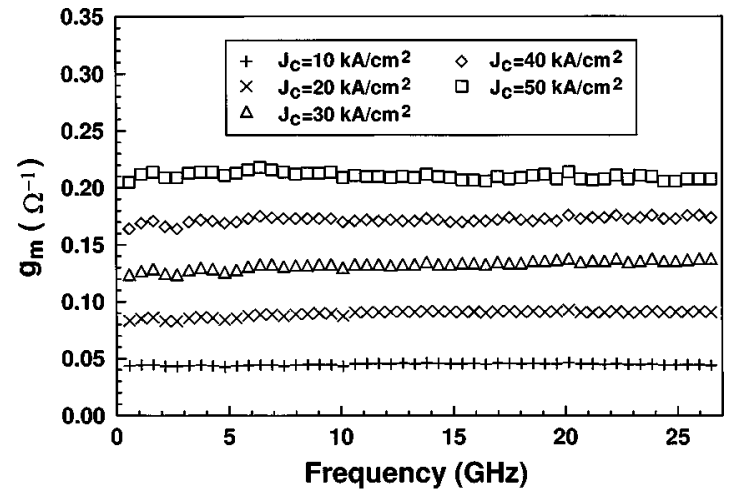

Fig. 6. The extracted quantities of the transconductance for the $2.4 \times 6 \mu \mathrm{m}^{2}$ emitter-area HBT biased at different collector current densities.

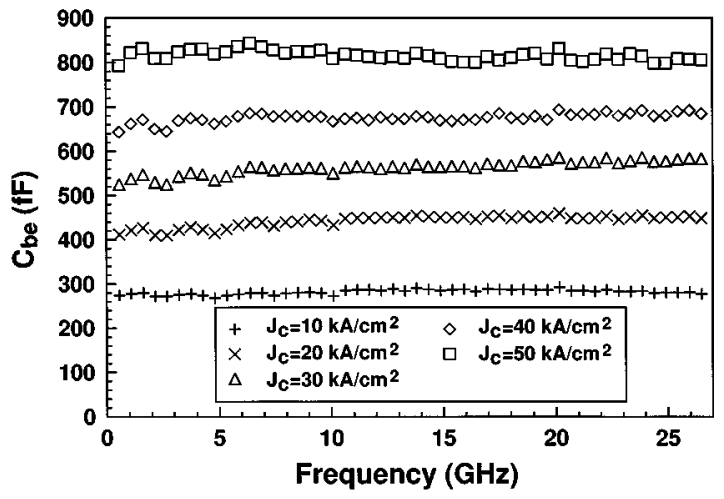

Fig. 7. The extracted quantities of the base-emitter capacitance for the $2.4 \times$ $6 \mu \mathrm{m}^{2}$ emitter-area HBT biased at different collector current densities.

\section{EXTRACTION OF EXTRINSIC ELEMENTS}

Although accurate extraction of the intrinsic elements including $C_{b c}^{t}, R_{b c}, g_{m}$, and $C_{b e}$ can be done without a precise quantity for $\beta$ and $X_{c j c}$, the remaining intrinsic elements $R_{b e}, R_{b}$ as well as the extrinsic elements have relatively close dependence on both parameters. In this section, the quantities of $\beta$ and $X_{c j c}$ are modified to better precision by exploiting their relations with the extrinsic elements. By re-examining (1)-(4), the extrinsic collector impedance can be derived as

$$
Z_{c}=R_{c}+j \omega L_{c}=\left(Z_{22}-Z_{12}\right)-\left(Z_{12}-Z_{21}\right) U
$$

where $U$ has been expressed in (24) as a function of $\beta$. Fig. 8 shows the calculated imaginary part of $Z_{c}$ against frequency for the HBT biased at $J_{\mathrm{c}}=20 \mathrm{kA} / \mathrm{cm}^{2}$. In principle, such a reactance should have an almost linear relation with frequency and its slope can be regarded as $2 \pi$ times the collector inductance. However, we have found that the reactance deviates from the linear relation at low frequencies with the presumed quantity of $\beta$ equal to 59 . We can seek the best linearity for the reactance by adjusting $\beta$ to 63 , which can be considered as a more acceptable quantity. Under this scheme, the collector resistances $\left(R_{c}\right)$ and inductances $\left(L_{c}\right)$ of the HBT biased at different collector current densities are extracted from (25) with the modified quantities of $\beta$, and their results are demonstrated in Figs. 9 and 10 , respectively. One can see that the extracted results can coincide with their extrinsic passive nature of weak bias dependence quite well.

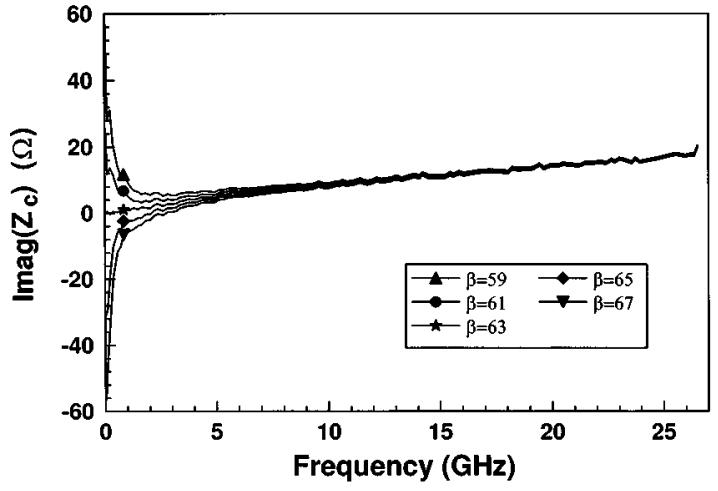

Fig. 8. Illustration of seeking the optimal quantity for the parameter $\beta$.

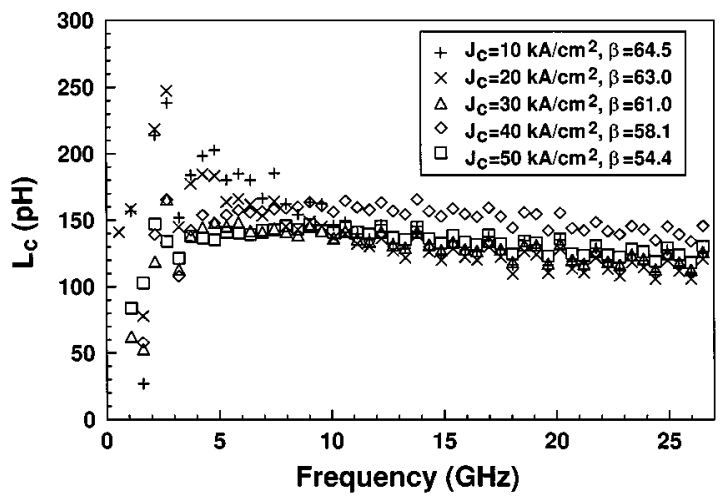

Fig. 9. The extracted quantities of the collector inductance for the $2.4 \times 6 \mu \mathrm{m}^{2}$ emitter-area HBT biased at different collector current densities.

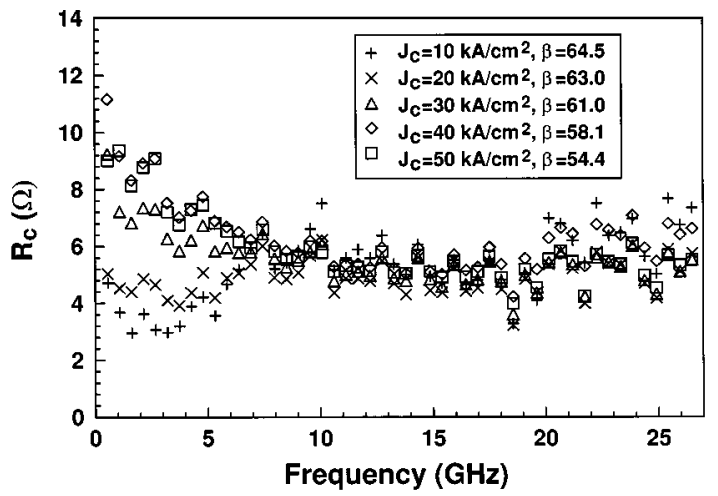

Fig. 10. The extracted quantities of the collector resistance for the $2.4 \times 6 \mu \mathrm{m}^{2}$ emitter-area HBT biased at different collector current densities.

Substituting (5) and (9) into (2), we can express the emitter inductance in the form

$$
\begin{aligned}
L_{e}=\omega^{-1} \operatorname{imag}\{ & \left.Z_{12}-\left[g_{m} e^{-j \omega \tau}(1+U)\right]^{-1}\right\} \\
& -R_{b} C_{b c}^{t}\left(1-X_{c j c}\right) \operatorname{real}\left\{\left(Z_{12}-Z_{21}\right)\right\} .
\end{aligned}
$$

Note that the above expression contains the base resistance $\left(R_{b}\right)$ and transconductance $\left(g_{m}\right)$, which have also been expressed as functions of $X_{c j c}$ in (20) and (21), respectively. In Fig. 11, the mean values of $L_{e}$ with respect to frequency have been calculated against $X_{c j c}$ under different collector current densities. It is observed that the emitter inductance has a close dependence on $X_{c j c}$. In general, for a fixed bias condition, the calculated emitter inductance increases with $X_{c j c}$ by varying from 0 to 1 , but appears positive only when $X_{c j c}$ exceeds a certain 


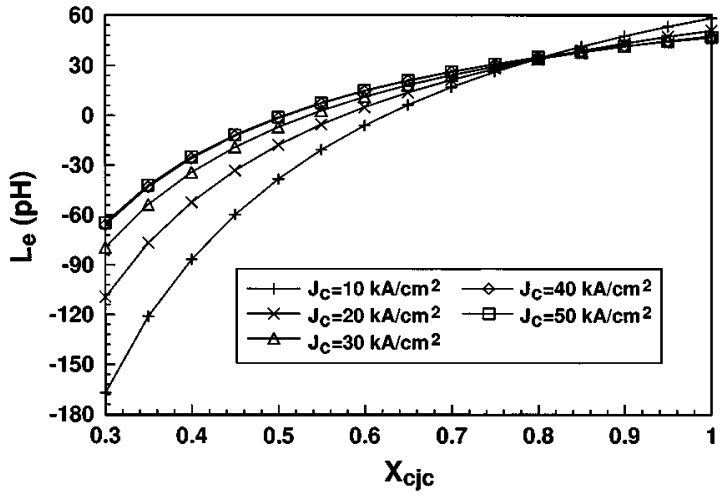

Fig. 11. Illustration of seeking the optimal quantities for the emitter inductance and the coefficient $X_{c j c}$.

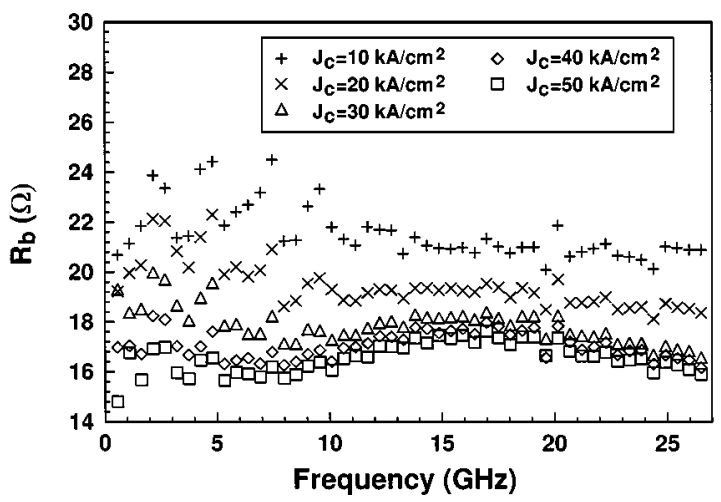

Fig. 12. The extracted quantities of the base resistance for the $2.4 \times 6 \mu \mathrm{m}^{2}$ emitter-area HBT biased at different collector current densities.

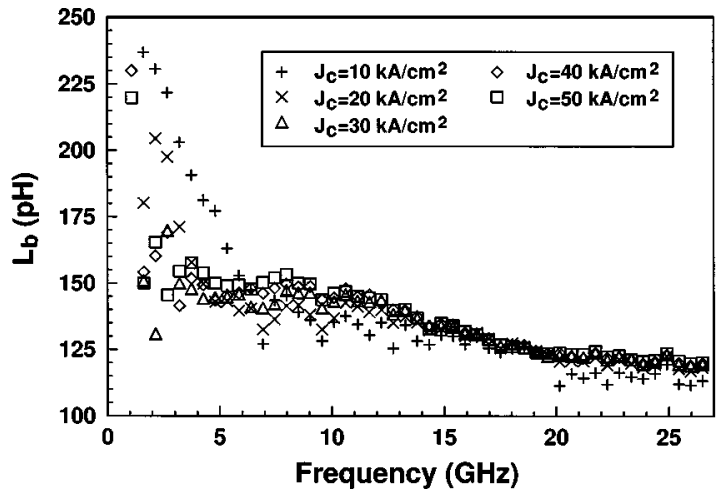

Fig. 13. The extracted quantities of the base inductance for the $2.4 \times 6 \mu \mathrm{m}^{2}$ emitter-area HBT biased at different collector current densities.

quantity. It can be also seen that all of the curves with different collector current densities intersect around $X_{c j c}=0.81$ and $L_{e}=34.4 \mathrm{pH}$, which can be regarded as the optimal quantities from the judgment of their bias-independent features. With the modified quantity of $X_{c j c}$, the base resistance has been re-calculated from (20) with results demonstrated in Fig. 12. It is observed that the base resistance tends to reduce at higher current densities. From (1), (2), (5), and (9), one can derive the base inductance in the form

$$
\begin{array}{r}
L_{b}=\omega^{-1} \operatorname{imag}\left\{R_{b} C_{b c}^{t}\left(Z_{21}-Z_{12}\right)(1+U)\left(\omega_{0} Q+j \omega X_{c j c}\right)\right. \\
\left.+\left(Z_{11}-Z_{12}\right)\right\} .
\end{array}
$$

The extracted results for the base inductance are shown in Fig. 13 and agree again with our expectation to have a weak bias dependence. The modified quantity of $X_{c j c}$ can be also used to recalculate the transconductance in (21) and subsequently the base-emitter resistance for better accuracy.

\section{CONCLUSION}

An extrinsic-inductance independent approach for direct extraction of HBT intrinsic elements has been presented. This approach starts with a formulation of the difference between two mutual impedance parameters that can exclude all the extrinsic inductances. In addition, the phase of such an impedance difference has been characterized by several parameters, which establish the fundamentals of the whole extraction. This makes the approach so unique to extract the intrinsic elements before extrinsic elements. The advantages include higher efficiency and stability in comparison with the other extraction techniques.

\section{ACKNOWLEDGMENT}

The authors would like to thank Dr. C. J. Wang, Advanced Wireless Semiconductor Company, Tainan, Taiwan, R.O.C., for providing many valuable HBT devices.

\section{REFERENCES}

[1] D. Costa, W. U. Liu, and J. S. Harris, "Direct extraction of the Al$\mathrm{GaAs} / \mathrm{GaAs}$ heterojunction bipolar transistor small-signal equivalent circuit," IEEE Trans. Electron Devices, vol. 38, pp. 2018-2024, Sept. 1991.

[2] C. J. Wei and J. C. M. Hwang, "Direct extraction of equivalent circuit parameters for heterojunction bipolar transistors," IEEE Trans. Microwave Theory Tech., vol. 43, pp. 2035-2039, Sept. 1995.

[3] Y. Gobert, P. J. Tasker, and K. H. Bachem, "A physical, yet simple smallsignal equivalent circuit for the heterojunction bipolar transistor," IEEE Trans. Microwave Theory Tech., vol. 45, pp. 149-153, Jan. 1997.

[4] M. Rudolph, R. Doerner, and P. Heymann, "Direct extraction of HBT equivalent-circuit elements," IEEE Trans. Microwave Theory Tech., vol. 47, pp. 82-84, Jan. 1999.

[5] S. Bousnina, P. Mandeville, A. B. Kouki, R. Surridge, and F. M. Ghannouchi, "A new analytical and broadband method for determining the HBT small-signal model parameters," in IEEE MTT-S Int. Microwave Symp. Dig., 2000, pp. 1397-1400.

[6] Y. Suh, E. Seok, J. H. Shin, B. Kim, D. Heo, A. Raghavan, and J. Laskar, "Direct extraction method for internal equivalent circuit parameters of HBT small-signal hybrid- $\pi$ model," in IEEE MTT-S Int. Microwave Symp. Dig., 2000, pp. 1401-1404.

[7] T.-S. Horng, J.-M. Wu, and H.-H. Huang, "An extrinsic-inductance independent approach for direct extraction of HBT intrinsic circuit parameters," in IEEE MTT-S Int. Microwave Symp. Dig., 2001, pp. 1761-1764.

[8] I. Getreu, Modeling the Bipolar Transistor. Beaverton, OR: Tekronix Inc., 1977.

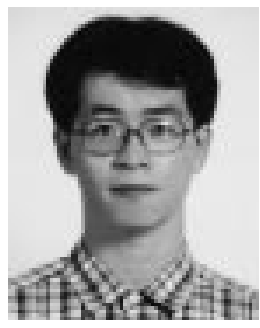

Tzyy-Sheng Horng (S'88-M'92) was born in Taichung, Taiwan, R.O.C, on December 7, 1963. He received the B.S.E.E. degree from the National Taiwan University, Taiwan, R.O.C., in 1985, and the M.S.E.E. and Ph.D. degrees from the University of California at Los Angeles, in 1990 and 1992, respectively.

$\mathrm{He}$ is currently an Associate Professor in the Department of Electrical Engineering, National Sun Yat-Sen University, Kaohsiung, Taiwan, R.O.C. His research interests are in the area of $\mathrm{RF}$ and microwave integrated circuits and packages. 


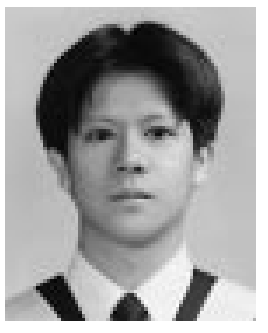

Jian-Ming Wu was born on November 13, 1974, in Kaohsiung, Taiwan, R.O.C. He received the B.S.E.E degree from the Yuan Ze University, Chungli, Taiwan, R.O.C., in 1997, the M.S.E.E. degree from the National Sun Yat-Sen University, Kaohsiung, Taiwan, R.O.C., in 2000, and is currently working toward the Ph.D. degree in electrical engineering at the National Sun Yat-Sen University.

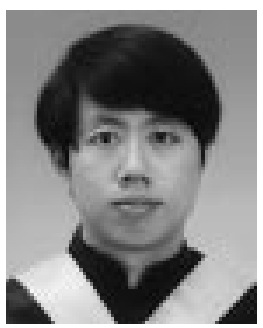

Hui-Hsiang Huang was born on April 9, 1972, in Pingtung, Taiwan, R.O.C. He received the B.S.E.E. degree from the Feng Chia University, Taichung, Taiwan, R.O.C., in 1995, and is currently working toward the M.S. degree in electrical engineering at the National Sun Yat-Sen University, Kaohsiung, Taiwan, R.O.C. 